\title{
Durvalumab activity in previously treated patients who stopped durvalumab without disease progression
}

\author{
Siddharth Sheth, ${ }^{1,2}$ Chen Gao, ${ }^{3}$ Nancy Mueller, ${ }^{2}$ Natasha Angra, ${ }^{2}$ Ashok Gupta, ${ }^{2}$ \\ Caroline Germa, ${ }^{2}$ Pablo Martinez, ${ }^{2}$ Jean-Charles Soria ${ }^{4}$
}

To cite: Sheth S, Gao C, Mueller N, et al. Durvalumab activity in previously treated patients who stopped durvalumab without disease progression. Journal for ImmunoTherapy of Cancer 2020;8:e000650. doi:10.1136/ jitc-2020-000650

- Additional material is published online only. To view please visit the journal online (http://dx.doi.org/10.1136/jitc2020-000650).

These results were presented in part at the Annual Meeting of the European Society for Medical Oncology, September 27, 2019 to October 1, 2019, Barcelona, Spain, oral presentation 11750.

Accepted 06 July 2020

Check for updates

(C) Author(s) (or their employer(s)) 2020. Re-use permitted under CC BY-NC. No commercial re-use. See rights and permissions. Published by BMJ.

For numbered affiliations see end of article.

Correspondence to Dr Siddharth Sheth; siddharth.sheth@med.unc.edu

\section{ABSTRACT}

Background Limited data exist on potential clinical benefit with anti-programmed cell death ligand-1 (PD-L1) retreatment in patients who stop initial therapy for reasons other than disease progression or toxicity and develop disease progression while off treatment.

Patients and methods NCT01693562 was a phase I/II study evaluating durvalumab monotherapy in advanced solid tumors. Patients benefiting from treatment were taken off durvalumab at 1 year per protocol and prospectively followed. At disease progression, they were eligible for durvalumab retreatment. Outcomes evaluated during retreatment included best overall response (BOR2), duration of response (DoR2), disease control rate (DCR2), and progression-free survival (PFS2).

Results Of 980 patients enrolled and treated with durvalumab $10 \mathrm{mg} / \mathrm{kg}$ every 2 weeks (Q2W) in the dose-expansion cohorts, 168 completed 1 year of initial durvalumab treatment with confirmed B0R1 of complete response in $20(11.9 \%)$, partial response (PR) in $84(50 \%)$, stable disease (SD) in 52 (31\%), and disease progression in $12(7.1 \%)$. All 168 patients stopped treatment and were eligible for retreatment at progression; 70 patients (41.7\%) representing 14 primary tumor types were retreated and response evaluable. Confirmed BOR2 was PR in 8 patients (11.4\%), SD in 42 (60.0\%), disease progression in 16 $(22.9 \%)$, and unevaluable in $4(5.7 \%)$. Median DoR2 was 16.5 months. DCR2 $\geq 24$ weeks (DCR2 24) was $47.1 \%$. PFS2 rate at 12 months was $34.2 \%$, and median PFS2 was 5.9 months. Median overall survival (OS2) was 23.8 months. Response rates, DCR2 24, and median DoR2 were generally greater in patients with high PD-L1 expression than those with low/negative expression. No new safety signals were observed during retreatment.

Conclusion Retreatment restored antitumor activity, resulting in high rates of durable disease control with an acceptable safety profile. This evidence supports retreatment of patients who stop anti-PD-L1 therapy for reasons other than progression or toxicity, and supports further investigation.

\section{INTRODUCTION}

A proportion of cancer patients treated with immune checkpoint inhibitors (ICIs), including inhibitors of programmed cell death-1 (PD-1) and its ligand (programmed cell death ligand-1; PD-L1), have meaningful and durable clinical responses. Research is now focusing on patient selection strategies and optimal duration of therapy, as well as reinitiating therapy in patients who stop treatment for reasons other than disease progression (eg, patient/provider decision or study protocol), who may maintain long-term responses. ${ }^{1-3}$ Rechallenging patients with ICIs after discontinuation due to adverse events (AEs) has an acceptable risk/benefit profile. ${ }^{4}$ The majority of prospective and retrospective data on ICI retreatment are in patients with non-small-cell lung cancer (NSCLG) or melanoma. Reinitiation of ipilimumab, an anti-CTLA-4 antibody, in 122 patients with advanced melanoma produced an objective response rate (ORR) of $23 \%$ (5.7\% complete responses (CRs) and $17.2 \%$ partial responses (PRs) ). ${ }^{5}$ An observational case series explored retreatment in 13 patients with advanced solid tumors who initially responded to nivolumab or pembrolizumab and discontinued at 1 year; of the eight $(61.5 \%)$ patients who progressed off treatment and were retreated, two had PRs and six had stable disease (SD). ${ }^{6}$ In the KEYNOTE-001 trial, one patient with NSCLC had a PR with retreatment after responding to initial therapy. Among 14 retreated patients in the KEYNOTE-010 trial, six had a PR. ${ }^{78}$ An analysis of 16 patients with advanced NSCLC who had survived at least 5 years after starting nivolumab treatment showed responses in the two patients who were retreated with an antiPD-1 agent after progression. ${ }^{9}$ In a prospective phase IIIb/IV safety study (CheckMate 153), limited clinical benefit was seen in patients with NSCLC who progressed after the initial 12-month nivolumab treatment period and underwent retreatment; the median duration of retreatment was 3.8 months. ${ }^{10}$ Furthermore, progression-free survival (PFS) was improved in patients who received continuous nivolumab therapy versus those who received an initial 12 months of treatment and were 
subsequently eligible for retreatment. The optimal treatment sequence and duration with ICIs remains unclear.

Here we report the effects of retreatment with durvalumab, a PD-L1 inhibitor, in patients who discontinued treatment without disease progression in Study 1108 , a phase I/II trial in advanced solid tumors (NCT01693562).

\section{PATIENTS AND METHODS}

The study design and initial results of this multicenter, open-label study have been described previously. ${ }^{11-13}$

This study was conducted in accordance with ethical principles that have their origin in the Declaration of Helsinki and are consistent with the International Council on Harmonization guidelines on Good Clinical Practice, any applicable laws and requirements, and any conditions required by a regulatory authority and/or Institutional Review Board/Independent Ethics Committee that has approved this study to be conducted in its territory. The study protocol was reviewed and approved by the Institutional Review Board or Ethics Committee of the participating centers, and informed consent was obtained.

The clinical data set analyzed here may be obtained in accordance with AstraZeneca's data sharing policy, which is described at https://astrazenecagrouptrials.pharmacm.com/ST/Submission/Disclosure

\section{Initial treatment period}

Eligibility criteria and treatment schedule are summarized in the online supplementary appendix. The primary objectives were safety and antitumor activity. Efficacy endpoints were ORR1, best overall response (BOR1), duration of response (DoR1), disease control rate (DCR1, defined as CR, PR and SD), PFS1 (from the first day of initial treatment to disease progression or death), and overall survival (OS1) (from the first day of initial treatment to death). Efficacy was assessed using Response Evaluation Criteria in Solid Tumors (RECIST) V.1.1.

\section{Retreatment period}

Per protocol, patients who maintained disease control (or otherwise demonstrated clinical benefit) stopped durvalumab after 1 year and entered follow-up. During the off-treatment period, patients received no systemic or local therapy. The frequency of clinical monitoring, which included clinical examinations and radiographic evaluations, was based on local/institutional standards of care. They were eligible for durvalumab retreatment $(10 \mathrm{mg} / \mathrm{kg}$ every two weeks $(\mathrm{Q} 2 \mathrm{~W}))$ at disease progression, continuing until confirmed disease progression or unacceptable toxicity, for up to 1 year.

Efficacy endpoints were ORR2, BOR2, DoR2, DCR2, PFS2 (from the first day of retreatment to disease progression or death), and OS2 (from the first day of retreatment to death). Safety endpoints were AEs, including treatment-related AEs (TRAEs) and serious AEs. Data were collected up to February 28, 2019. The main objectives
Table 1 Demographics and baseline clinical characteristics of response-evaluable patients at initiation of retreatment

\begin{tabular}{|c|c|}
\hline Characteristic & All patients $(n=70)$ \\
\hline Median age (range), years & 61.0 (31 to 87$)$ \\
\hline \multicolumn{2}{|l|}{ Sex, n (\%) } \\
\hline Male & $42(60.0)$ \\
\hline Female & $28(40.0)$ \\
\hline \multicolumn{2}{|l|}{ Race, n (\%) } \\
\hline Asian & $13(18.6)$ \\
\hline Black & $4(5.7)$ \\
\hline White & $43(61.4)$ \\
\hline Other & $2(2.9)$ \\
\hline Not reported & $8(11.4)$ \\
\hline \multicolumn{2}{|c|}{ Eastern Cooperative Oncology Group performance status, n (\%) } \\
\hline 0 & $33(47.1)$ \\
\hline 1 & $37(52.9)$ \\
\hline \multicolumn{2}{|l|}{ Smoking history, n (\%) } \\
\hline Current & $4(5.8)$ \\
\hline Former & $40(58.0)$ \\
\hline Never & $25(36.2)$ \\
\hline Not reported & $1(1.4)$ \\
\hline \multicolumn{2}{|l|}{ Tumor type, n (\%) } \\
\hline $\mathrm{HCC}$ & $5(7.1)$ \\
\hline HNSCC & $6(8.6)$ \\
\hline MSI-high cancers & $12(17.1)$ \\
\hline NSCLC & $21(30.0)$ \\
\hline UC & $8(11.4)$ \\
\hline Other tumors* & $18(25.7)$ \\
\hline \multicolumn{2}{|c|}{ Number of systemic prior treatments, $\mathrm{n}(\%)$} \\
\hline 1 & $22(31.4)$ \\
\hline 2 & $13(18.6)$ \\
\hline$\geq 3$ & $28(40.0)$ \\
\hline Not reported & $7(10.0)$ \\
\hline \multicolumn{2}{|l|}{ PD-L1 status, $n(\%)^{\dagger}$} \\
\hline High & $22(62.9)$ \\
\hline Low & $11(31.4)$ \\
\hline Unknown & $2(5.7)$ \\
\hline Not reported & $35(50.0)$ \\
\hline \multicolumn{2}{|l|}{ Previous IO history, n (\%) } \\
\hline Yes & $2(2.9)$ \\
\hline No & $68(97.1)$ \\
\hline Mean BMI $\left(\mathrm{kg} / \mathrm{m}^{2}\right)$ & 26.73 \\
\hline \multicolumn{2}{|l|}{ Liver metastasis, $\mathrm{n}(\%)$} \\
\hline Yes & $15(21.4)$ \\
\hline No & $55(78.6)$ \\
\hline
\end{tabular}

*Advanced melanoma $(n=2)$, gastrointestinal cancer $(n=1)$, human papilloma virus-positive cervical cancer $(n=3)$, nasopharyngeal cancer $(n=2)$, ovarian cancer $(n=2)$, pancreatic cancer $(n=1)$, small-cell lung cancer $(n=4)$, triple-negative breast cancer $(n=2)$, and uveal melanoma $(n=2)$. $(\mathrm{n}=1)$, small-cell lung cancer $(\mathrm{n}=4)$, triple-negative breast cancer $(\mathrm{n}=2)$, and uveal melanoma
tOnly available in patients with UC, HNSCC and NSCLC tumors (based on guidelines for individual tumor indications).

BMI, body mass index; HCC, hepatocellular carcinoma; HNSCC, head and neck squamous cell BMI, body mass index; HCC, hepatocellular carcinoma; HNSCC, head and neck squamous cell
carcinoma; IO, immuno-oncology; MSI, microsatellite instability; NSCLC, non-small-cell lung cancer; PD-L1, programmed cell death ligand-1; UC, urothelial carcinoma.

were to characterize the BOR2 per RECIST V.1.1, evaluate DCR2 at 24 weeks (DCR2 24), and determine the 12-month PFS2 rate and median OS2. Statistical analyses are described in the online supplementary appendix. 


\section{Evaluation of PD-L1 status}

Tumor PD-L1 status was only assessed in patients with urothelial carcinoma (UC), head and neck squamous cell carcinoma (HNSCC), and NSCLC. Expression was evaluated prior to initial durvalumab treatment using the SP263 immunohistochemical (IHC) assay (Ventana Medical Systems, Inc). High expression was defined as $\geq 25 \%$ of tumor cell membranes (tumor or immune cells for UC) staining positive for PD-L1 at any intensity. PD-L1 low/negative was defined as $<25 \%$ of tumor cells (both tumor cells and immune cells for UC) staining for PD-L1. Expression was not measured at retreatment.

\section{RESULTS \\ Characteristics of patients who received durvalumab retreatment}

A total of 1022 patients were enrolled in Study 1108 and received treatment with durvalumab, 980 at an initial dose of $10 \mathrm{mg} / \mathrm{kg}$ Q2W. Among these 980 patients, 812 $(82.9 \%)$ had $<1$ year of treatment and therefore were not eligible for retreatment. The remaining 168 patients (17.1\%) completed 1 year of treatment and discontinued per protocol. During initial treatment, BOR1 was CR in 20 patients $(11.9 \%), \mathrm{PR}$ in $84(50.0 \%)$, SD in $52(31.0 \%)$, and disease progression in $12(7.1 \%)$. Median OS1 was 48.9 months (includes initial treatment and retreatment periods). Of the 168 eligible patients, 97 (57.7\%) progressed while off treatment but were not retreated with durvalumab (online supplementary table 1 ). The remaining 71 patients (42.3\%) received durvalumab retreatment at disease progression.

\section{Characteristics of retreated patients during initial durvalumab treatment}

Of the 71 retreated patients, one patient with glioblastoma was non-evaluable by RECIST for initial treatment and was excluded from further evaluation. For the remaining 70 patients, BOR1 was CR in four $(5.7 \%)$, PR in $35(50.0 \%)$, SD in $25(35.7 \%)$, and progressive disease in six $(8.6 \%)$. Of the six patients with progressive disease by RECIST, four had pseudoprogression and had PR by iRECIST per investigator evaluation. The two remaining patients had true progressive disease but were maintained on durvalumab based on investigator decision.

\section{Baseline characteristics at retreatment}

Fourteen tumor types were represented, most commonly NSCLC, microsatellite instability (MSI)-high cancers, UC, HNSCC, and hepatocellular carcinoma (HCC). The group sizes for the remaining nine tumor types were too small for individual analysis; therefore, they were pooled together as Other tumors. Demographic and baseline characteristics are summarized in table 1. Characteristics of the 70 retreated patients were similar to those of the full Study 1108 population as well as the 168 patients eligible for retreatment. Patients undergoing retreatment were heavily pretreated, though only two had prior ICI

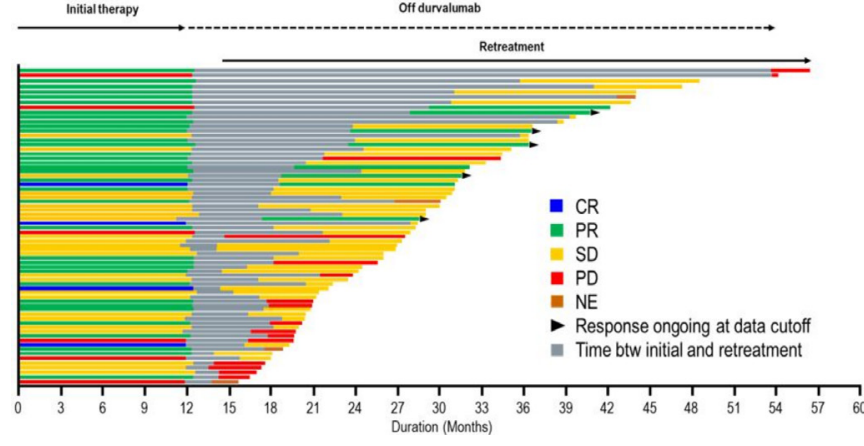

Figure 1 Response during initial treatment, time off treatment, and response during retreatment. Median time off treatment was 6.8 months. Response to retreatment was not evaluable per RECIST in four patients; they are depicted as NE. btw, between; CR, complete response; NE, non-evaluable; PR, partial response; RECIST, Response Evaluation Criteria in Solid Tumors; SD, stable disease; PD, progressive disease.

exposure. PD-L1 expression was measured in 35 patients (50\%): it was high in $22(62.9 \%)$, low/negative in 11 $(31.4 \%)$, and unknown in two $(5.7 \%)$.

\section{Retreatment response}

Median time off treatment following initial treatment was 6.8 months (figure 1). Eight responses occurred during retreatment (ORR2: $11.4 \%, 95 \%$ CI: 5.1 to 21.3); all were PRs (table 2). In addition, 42 patients $(60.0 \%)$ had SD, including two (2.9\%) unconfirmed PRs. Responses were ongoing in five patients at data cut-off; the longest response exceeded 25.1 months in a patient with NSCLC. Twenty-two of the 70 patients $(31.4 \%)$ completed 1 year of retreatment.

Table 2 Clinical response to initial treatment and retreatment with durvalumab

\begin{tabular}{lll}
\hline Response & $\begin{array}{l}\text { Initial } \\
\text { treatment } \\
(\mathbf{n}=\mathbf{7 0})\end{array}$ & $\begin{array}{l}\text { Retreatment } \\
(\mathbf{n}=70)\end{array}$ \\
\hline $\begin{array}{l}\text { Best overall response, } \mathrm{n}(\%) \\
\quad \text { Complete response }\end{array}$ & $4(5.7)$ & 0 \\
\hline $\begin{array}{l}\text { Partial response } \\
\text { Stable disease }\end{array}$ & $35(50.0)$ & $8(11.4)$ \\
\hline $\begin{array}{l}\text { Unconfirmed partial } \\
\text { response }\end{array}$ & $25(35.7)$ & $42(60.0)$ \\
\hline $\begin{array}{l}\text { Disease progression } \\
\text { Non-evaluable }\end{array}$ & $6(2.9)$ & $2(2.9)$ \\
\hline $\begin{array}{l}\text { Median time to response, } \\
\text { months }\end{array}$ & 0 & $16(22.9)$ \\
\hline $\begin{array}{l}\text { Median duration of response, } \\
\text { months }\end{array}$ & 14.8 & $4(5.7)$ \\
\hline DCR $\geq 24$ weeks, \% & 82.9 & 4.3 \\
\hline PFS rate at 12 months, \% & 71.0 & 16.5 \\
\hline Median OS, months & 48.9 & 34.1 \\
\hline
\end{tabular}

DCR, disease control rate; OS, overall survival; PFS, progression-free survival. 
Patients with an objective response (CR or PR) to initial durvalumab therapy experienced a longer time off treatment until disease progression compared with those without an objective response (8.5 vs 6.0 months, $\mathrm{p}=0.03$ ). However, objective responses during initial treatment were not associated with objective responses or longer times to disease progression during retreatment. Only 5/8patients had objective responses at both initial treatment and retreatment. Two patients with SD on initial treatment developed a PR on retreatment, both of which were ongoing at the time of data cutoff. Finally, one patient with pseudoprogression (PR by iRECIST) on initial treatment had a PR with retreatment. The other three patients with pseudoprogression (PR by iRECIST) on initial treatment had disease progression on retreatment.

\section{Response and survival with initial treatment and retreatment}

The median time to response was shorter with initial treatment compared with retreatment ( 2.7 vs 4.3 months; table 2). However, the median duration of response was similar (14.8 vs 16.5 months). Retreatment DCR2 24 and 12-month PFS2 rates were about half of those observed with initial treatment $(47.1 \%$ vs $82.9 \%$, and $34.2 \%$ vs $71.0 \%$, respectively). Median OS2 with retreatment was 23.8 months.

\section{Benefit of durvalumab retreatment by tumor types and other characteristics}

Objective responses occurred in multiple tumor indications. While no responses were seen in the MSI-high tumor group, $91.7 \%$ experienced SD. Antitumor activity was observed in all tumor types (figure 2, online supplementary figure 1). The DCR2 24 was $47.1 \%$ overall, ranging from $33.3 \%$ in NSCLC and HNSCC to $61.1 \%$ in Other tumors (online supplementary table 2). At 12 months, PFS2 rates ranged from $25.0 \%$ (HCC) to $62.5 \%$ (UC). Among the 35 patients with available PD-L1 evaluations, ORR2 (22.7\% vs 9.1\%), median DoR2 (16.5 vs

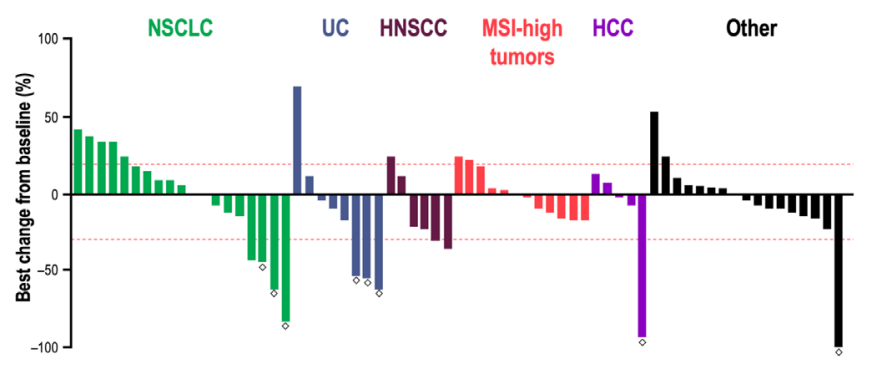

$\diamond$ Confirmed PR

Figure 2 Retreatment resulting in antitumor activity across all tumor indications. HCC, hepatocellular carcinoma; HNSCC, head and neck squamous cell carcinoma; MSI, microsatellite instability; NSCLC, non-small-cell lung cancer; PR, partial response; UC, urothelial cancer.
Table 3 AEs during initial treatment and retreatment with durvalumab

\begin{tabular}{|c|c|c|}
\hline AEs & $\begin{array}{l}\text { Initial } \\
\text { treatment } \\
(n=70)\end{array}$ & $\begin{array}{l}\text { Retreatment } \\
(n=70)\end{array}$ \\
\hline $\begin{array}{l}\geq 1 \text { treatment-emergent } A E \text {, } \\
n(\%)\end{array}$ & $69(98.6)$ & $64(91.4)$ \\
\hline $\begin{array}{l}\geq 1 \text { grade } 3 \text { or } 4 \text { treatment- } \\
\text { emergent } A E, n(\%)\end{array}$ & $24(34.3)$ & $31(44.3)$ \\
\hline >1 TRAE, n (\%) & $53(75.7)$ & $28(40.0)$ \\
\hline$\geq 1$ grade 3 or 4 TRAE, $n(\%)$ & $2(2.9)$ & $4(5.7)$ \\
\hline $\begin{array}{l}\geq 1 \text { TRAE leading to } \\
\text { durvalumab discontinuation }\end{array}$ & 0 & $2(2.9)$ \\
\hline$\geq 1$ serious TRAE, $\mathrm{n}(\%)$ & $2(2.9)$ & $2(2.9)$ \\
\hline Deaths & 0 & 0 \\
\hline
\end{tabular}

TRAE, treatment-related adverse event.

7.2 months), and DCR2 24 (54.5\% vs $9.1 \%$ ) were superior in those with PD-L1 high expression than those with low/negative expression (online supplementary table 3). Baseline liver metastases at retreatment were not predictive for response.

\section{Safety of retreatment}

Retreatment was well tolerated. The majority of patients experienced $\geq 1$ treatment-emergent $\mathrm{AE}$ (table 3), most commonly diarrhea $(27.1 \%)$, fatigue $(21.4 \%)$, cough $(21.4 \%)$, decreased appetite $(20.0 \%)$, and nausea $(17.1 \%)$. Forty per cent of patients had TRAEs. The incidence of grade $3 / 4$ TRAEs was $5.7 \%$; they were increased alanine aminotransferase, hyperglycemia, pancreatitis, and pneumonitis (1.4\% each). Serious TRAEs occurred in two patients $(2.9 \%)$ : abdominal pain and pancreatitis in one and pneumonitis in the other; both discontinued treatment. There were no treatment-related deaths.

\section{DISCUSSION}

This study demonstrates that retreatment with durvalumab can be safe and effective in patients who stop treatment and progress while off therapy. Of the 70 retreated patients, more than $70 \%$ experienced clinical benefit (11.4\% with PR and $60.0 \%$ with SD). This suggests that retreatment restored antitumor activity in a majority of patients, resulting in high rates of durable disease control with an acceptable safety profile. Despite its retrospective design, the study had multiple strengths, including the analysis of the largest retreatment cohort in this setting, the inclusion of diverse tumor types, and treatment homogeneity.

There are no established guidelines for optimal duration of initial therapy with ICIs. In clinical practice and in many research protocols, patients are treated through the first evidence of disease progression to account for tumor 
flare (pseudoprogression) and to provide adequate time for ICI activity to become apparent, which may take longer than with conventional cytotoxic chemotherapies. In patients who are benefiting from ICIs, treatment may continue for 2 years or even longer. ${ }^{14}{ }^{15}$ Patients and providers hesitate to discontinue treatment without disease progression. However, indefinite treatment raises concerns about long-term safety, costs, and quality of life. The outcomes of this study permit a more informed decision process.

Information on retreatment with ICIs is limited, with previous studies largely conducted in NSCLC or melanoma. In a study of pembrolizumab for metastatic melanoma, patients with CRs discontinued therapy after $>6$ months. Four patients developed disease recurrence and were retreated with pembrolizumab; one had CR, one had SD, and the remaining two had PD. ${ }^{1}$ Similarly, in a retrospective study of anti-PD-1 monotherapy for metastatic melanoma, 5/34 patients responded to retreatment. ${ }^{16}$ In a rechallenge series of 144 patients with NSCLC who discontinued initial ICI treatment, retreatment PFS was longer in those who discontinued due to toxicity or clinical decision than in those who discontinued due to disease progression. ${ }^{17}$ In a retrospective study, patients with NSCLC who had SD or progressive disease in response to atezolizumab or durvalumab were switched to nivolumab or pembrolizumab; among the 13 evaluable patients, four had SD and nine had disease progression with a short PFS. ${ }^{18}$ The differences between these data and the current study indicate that retreatment should be reserved for patients who benefit from initial ICI therapy.

In the present study, ORR2 was significantly lower than ORR1 $(11.4 \%$ vs $55.7 \%, \mathrm{p}<0.01)$. Despite small numbers, objective responses were observed in UC (3/8) and NSCLC (3/21), which are immunotherapy-sensitive tumors. ${ }^{19} 20$ Furthermore despite low ORR2, 34/66 patients $(51.5 \%)$ with evaluable scans had a decrease in on-treatment tumor volume compared with baseline (figure 2), indicative of meaningful clinical benefit.

The rates of response and disease stability, and the duration of clinical benefit were comparable to those of second-line or later-line chemotherapy regimens following immunotherapy. ${ }^{21}{ }^{22}$ The high rates of durable disease control (DCR2 24) and PFS2 suggest the possibility of immune rescue and restoration of immune memory. Importantly, differences were observed based on tumor types and PD-L1 expression. For example, $>90 \%$ of patients with MSI-high tumors had SD, which exceeded 24 weeks in $58.3 \%$. This is likely due to both tumor-cell intrinsic genetic and immune-related features. MSI-high tumors have increased tumor-infiltrating lymphocyte density relative to microsatellite-stable tumors, ${ }^{23}$ and higher rates of CD8+ T-cell infiltration, PD-L1 expression, and IFN $\gamma$ production. ${ }^{24-26}$ Similarly with PFS2, the highest 12-month rate was in UC $(62.5 \%)$, which is particularly immunotherapysensitive at high grades. ${ }^{19}$ The lowest rate was in HNSCC $(0 \%)$, which may not be a disease-specific phenomenon but rather explained by tumor-specific factors such as human papilloma virus and smoking status.

Patients with PD-L1 high expression at baseline demonstrated higher ORR2 and DCR2 24, and longer median DoR2 than patients with low/negative expression. Additional research is needed on other potential predictive biomarkers such as tumor mutational burden, oncogenic driver mutations, and circulating DNA mutant allele fractions.

The durability of disease control with durvalumab retreatment in Study 1108, evidenced by a DCR2 24 of $47.1 \%$ across the 14 primary tumor types, represents a clinically meaningful benefit in a heavily pretreated patient population. Most randomized trials involving ICI retreatment are in NSCLC and melanoma, with relatively few patients in each study entering retreatment. Furthermore, they often focus more on disease control rate than on its duration, giving a limited picture of longer-term therapeutic benefits. In KEYNOTE-010, a DCR2 of 78.6\% was recorded in 14 patients with advanced NSCLC who were retreated with pembrolizumab. ${ }^{8}$ In KEYNOTE-024, the DCR2 was $70 \%$ in a treatment-naive patient population with NSCLC. ${ }^{27}$ In the present study, DCR2 was $52.4 \%$ in the 21 patients with NSCLC and DCR2 24 was 33.3\%, indicating that long-term clinical benefit may be possible for many patients who experience disease control with durvalumab retreatment. Among the 13 patients with metastatic melanoma who underwent retreatment with pembrolizumab in the KEYNOTE-006 study, the DCR2 was $76.9 \% .^{28}$ Crucially, only $2 / 71$ patients in the retreatment population of Study 1108 had melanoma, a tumor with high sensitivity to immunotherapy; therefore, only limited inference can be made through comparison with studies focusing solely on patients with melanoma.

The durability of clinical benefit varied among patients who completed 1 year of initial durvalumab treatment. No patients remained progression-free at the data cutoff for the current analysis, regardless of whether they were retreated. However, among the patients who were retreated, the interval time without progression differed greatly-some patients remained off treatment for over 1 year and had an OS of over 3 years from the start of initial therapy (figure 1). Of the retreated patients, five had a BOR2 of PR that was ongoing at the data cut-off, with some lasting for over 1 year.

The study had important limitations. Only 168/1022 patients $(16.4 \%)$ in the initial durvalumab treatment population were eligible for retreatment, suggesting that the results only apply to a select patient population. Small patient numbers per individual tumor type precludes tumor-specific recommendations. Much remains unknown about the complex factors that influence tumor immunogenicity and checkpoint inhibitor efficacy in retreatment. Patients in the present study were limited to a maximum of 1 year of retreatment with durvalumab; however, this stipulation has not been included in subsequent clinical trials of this agent. Importantly, there are currently no formal guidelines 
regarding the appropriate duration of initial treatment or retreatment with ICIs. This study was retrospective and future research should prospectively evaluate clinical benefit using a control arm.

The encouraging safety and efficacy data, particularly the high DCR2 24 rate and the long DoR2, support durvalumab retreatment in patients who progress after a planned treatment stop following initial benefit. Future research should explore mechanisms of retreatment activity and discontinuation after shorter initial treatment periods.

\section{Author affiliations}

${ }^{1}$ Division of Medical Oncology, Department of Medicine, University of North Carolina at Chapel Hill, Chapel Hill, North Carolina, USA

${ }^{2}$ Clinical Development Oncology, Oncology Research and Development,

AstraZeneca, Gaithersburg, Maryland, USA

${ }^{3}$ Biometrics Oncology, Oncology Research and Development, AstraZeneca, Gaithersburg, Maryland, USA

${ }^{4}$ Oncology Research and Development, AstraZeneca, Gaithersburg, Maryland, USA

Acknowledgements The authors would like to thank the patients, their families and caregivers, trial nurses, data managers, and all study investigators for their contributions to study conduct. The authors also thank Rama Arja, MS, Sai Buragadda, MS, Sangeeta Anandula, MS, and Ramesh Karuppsamy, MS, of AstraZeneca Biostatistics; and Jacob Lacayo, MS, and Mary Benson, MS, of AstraZeneca Clinical Operations.

Contributors Acquisition of data: SS, CGa, NM, and NA. Analysis and interpretation of data: SS, CGa, and PM. Review and revision of the manuscript: SS, CGa, NM, NA, AG, CGe, PM, and JCS. Writing, review, and/or revision of the manuscript: SS, CGa, NM, NA, AG, CGe, PM, and JCS. Conception and design: SS and PM. Development of methodology: SS, CGa, NA, and PM.

Funding Study 1108 (NCT01693562) was funded by AstraZeneca. This analysis was supported by the 2018 American Association for Cancer Research (AACR)/ AstraZeneca Immuno-oncology Research Training Fellowship, Grant Number 18-40-12-SHET. Medical writing support was provided by James Holland and Susanne Gilbert of Cirrus Communications (Macclesfield, UK) and was funded by AstraZeneca (Gaithersburg, Maryland, USA).

Competing interests SS received travel support from AstraZeneca relating to this study. CGa, NM, NA, AG, CGe, PM, and JCS are employees of and stockholders in AstraZeneca. Over the last 5 years, JCS has received consultancy fees from AstraZeneca, Astex, Clovis, GSK, GamaMabs, Lilly, MSD, Mission Therapeutics, Merus, Pfizer, Pharma Mar, Pierre Fabre, Roche/Genentech, Sanofi, Servier, Symphogen, and Takeda. JCS has been a full-time employee of AstraZeneca since September 2017. He is a shareholder of AstraZeneca and Gritstone.

\section{Patient consent for publication Not required.}

Ethics approval The study was performed in accordance with ethical principles that have their origin in the Declaration of Helsinki and are consistent with the International Conference on Harmonization/Good Clinical Practice and applicable regulatory requirements. The protocol was approved by Institutional Review Boards (IRBs)/Independent Ethics Committees (IECs) at each center. Patients provided written informed consent to participate.

Provenance and peer review Not commissioned; externally peer reviewed.

Data availability statement Data are available upon reasonable request. The clinical data set analyzed during the current study is available at clinicaltrials.gov, https://clinicaltrials.gov/ct2/show/NCT01693562. The clinical data set analyzed here may be obtained in accordance with AstraZeneca's data sharing policy, which is described at https://astrazenecagrouptrials.pharmacm.com/ST/Submission/ Disclosure.

Open access This is an open access article distributed in accordance with the Creative Commons Attribution Non Commercial (CC BY-NC 4.0) license, which permits others to distribute, remix, adapt, build upon this work non-commercially, and license their derivative works on different terms, provided the original work is properly cited, appropriate credit is given, any changes made indicated, and the use is non-commercial. See http://creativecommons.org/licenses/by-nc/4.0/.

\section{REFERENCES}

1 Hamid O, Robert C, Daud A, et al. Five-year survival outcomes for patients with advanced melanoma treated with pembrolizumab in KEYNOTE-001. Ann Oncol 2019;30:582-8.

2 Gettinger SN, Horn L, Gandhi L, et al. Overall survival and long-term safety of nivolumab (anti-programmed death 1 antibody, BMS936558, ONO-4538) in patients with previously treated advanced non-small-cell lung cancer. J Clin Oncol 2015;33:2004-12.

3 Schadendorf D, Wolchok JD, Hodi FS, et al. Efficacy and safety outcomes in patients with advanced melanoma who discontinued treatment with nivolumab and ipilimumab because of adverse events: a pooled analysis of randomized phase II and III trials. J Clin Oncol 2017;35:3807-14.

4 Simonaggio A, Michot JM, Voisin AL, et al. Evaluation of readministration of immune checkpoint inhibitors after immunerelated adverse events in patients with cancer. JAMA Oncol 2019;5:1310-7.

5 Lebbé C, Weber JS, Maio M, et al. Survival follow-up and ipilimumab retreatment of patients with advanced melanoma who received ipilimumab in prior phase II studies. Ann Oncol 2014;25:2277-84.

6 Bernard-Tessier A, Baldini C, Martin P, et al. Outcomes of long-term responders to anti-programmed death 1 and anti-programmed death ligand 1 when being rechallenged with the same anti-programmed death 1 and anti-programmed death ligand 1 at progression. Eur $J$ Cancer 2018;101:160-4.

7 Garon EB, Hellmann MD, Rizvi NA, et al. Five-year overall survival for patients with advanced non-small-cell lung cancer treated with pembrolizumab: results from the phase I KEYNOTE-001 study. J Clin Oncol 2019;37:2518-27.

8 Herbst RS, Garon EB, Kim D-W, et al. Long-term outcomes and retreatment among patients with previously treated, programmed death-ligand 1-positive, advanced non-small-cell lung cancer in the KEYNOTE-010 study. J Clin Oncol 2020;38:1580-90.

9 Gettinger S, Horn L, Jackman D, et al. Five-year follow-up of nivolumab in previously treated advanced non-small-cell lung cancer: results from the CA209-003 study. J Clin Oncol 2018;36:1675-84.

10 Spigel DR, McLeod M, Hussein MA, et al. Randomized results of fixed-duration (1-yr) vs continuous nivolumab in patients (pts) with advanced non-small cell lung cancer (NSCLC). Ann Oncol 2017;28(suppl 5). 10.1093/annonc/mdx380

11 Antonia SJ, Balmanoukian A, Brahmer J, et al. Clinical activity, tolerability, and long-term follow-up of durvalumab in patients with advanced NSCLC. J Thorac Oncol 2019;14:1794-806.

12 Segal NH, Ou S-HI, Balmanoukian A, et al. Safety and efficacy of durvalumab in patients with head and neck squamous cell carcinoma: results from a phase I/II expansion cohort. Eur J Cancer 2019;109:154-61.

13 Powles T, O'Donnell PH, Massard C, et al. Efficacy and safety of durvalumab in locally advanced or metastatic urothelial carcinoma: updated results from a phase 1/2 open-label study. JAMA Oncol 2017:3:e172411.

14 Saba NF, Blumenschein G Jr, Guigay J, et al. Nivolumab versus investigator's choice in patients with recurrent or metastatic squamous cell carcinoma of the head and neck: efficacy and safety in CheckMate 141 by age. Oral Oncol 2019;96:7-14.

15 Kluger HM, Chiang V, Mahajan A, et al. Long-term survival of patients with melanoma with active brain metastases treated with pembrolizumab on a phase II trial. J Clin Oncol 2019;37:52-60.

16 Betof Warner A, Palmer JS, Shoushtari AN, et al. Responders to antiPD1 therapy: long-term outcomes and responses to retreatment in melanoma (mel). J Clin Oncol 2019;37:9513.

17 Gobbini E, Toffart AC, Perol M. Immune checkpoint inhibitor (ICPI) re-challenge: outcomes analysis in a French national cohort of nonsmall-cell lung cancer (NSCLC) patients. Presented at WCLC; 8 Sep, Barcelona, Spain, 2019:MA07.05.

18 Fujita K, Yamamoto Y, Kanai O, et al. Retreatment with anti-PD-1 antibody in non-small cell lung cancer patients previously treated with anti-PD-L1 antibody. Thorac Cancer 2020;11:15-18.

19 Hodgson A, Liu SK, Vesprini D, et al. Basal-subtype bladder tumours show a 'hot' immunophenotype. Histopathology 2018;73:748-57.

20 Maleki Vareki S. High and low mutational burden tumors versus immunologically hot and cold tumors and response to immune checkpoint inhibitors. J Immunother Cancer 2018;6:157.

21 Corral J, Majem M, Rodríguez-Abreu D, et al. Efficacy of nintedanib and docetaxel in patients with advanced lung adenocarcinoma treated with first-line chemotherapy and second-line immunotherapy in the nintedanib NPU program. Clin Trans/ Oncol 2019;21:1270-9.

22 Lam JM, Liu WK, Powles T, et al. Single-centre experience of patients with metastatic urothelial cancer treated with chemotherapy following immune checkpoint inhibition. Eur Urol Oncol 2019. doi:10.1016/j.euo.2019.03.001. [Epub ahead of print: 09 Apr 2019]. 
23 Fabrizio DA, George TJ Jr, Dunne RF, et al. Beyond microsatellite testing: assessment of tumor mutational burden identifies subsets of colorectal cancer who may respond to immune checkpoint inhibition. $J$ Gastrointest Oncol 2018:9:610-7.

24 De Smedt L, Lemahieu J, Palmans S, et al. Microsatellite instable vs stable colon carcinomas: analysis of tumour heterogeneity, inflammation and angiogenesis. Br J Cancer 2015;113:500-9.

25 Morihiro T, Kuroda S, Kanaya N, et al. PD-L1 expression combined with microsatellite instability/CD8+ tumor infiltrating lymphocytes as a useful prognostic biomarker in gastric cancer. Sci Rep 2019;9:4633.
26 Wang $\mathrm{H}$, Wang $\mathrm{X}, \mathrm{Xu} \mathrm{L}$, et al. Analysis of the transcriptomic features of microsatellite instability subtype colon cancer. BMC Cancer 2019;19:605.

27 Reck M, Rodríguez-Abreu D, Robinson AG, et al. OA14.01 KEYNOTE-024 3-year survival update: pembrolizumab vs platinumbased chemotherapy for advanced non-small-cell lung cancer. $J$ Thorac Oncol 2019;14:S243.

28 Robert C, Ribas A, Schachter J, et al. Pembrolizumab versus ipilimumab in advanced melanoma (KEYNOTE-006): post-hoc 5-year results from an open-label, multicentre, randomised, controlled, phase 3 study. Lancet Oncol 2019;20:1239-51. 